\title{
Escala de Generatividade do Casal: Tradução, Adaptação e Estudo das Propriedades Psicométricas da Versão Portuguesa
}

\author{
Couple Generativity Scale: Translation, Adaptation and Study of Psychometric \\ Properties of the Portuguese Version
}

\author{
Susana Costa-Ramalho ${ }^{1}$, Alexandra Marques-Pinto ${ }^{2}$, Maria Teresa Ribeiro ${ }^{3}$ e Marta Pedro ${ }^{4}$
}

\begin{abstract}
Resumo
A importância da conjugalidade na vida adulta justifica o estudo de fatores que possam contribuir para a sua qualidade. Este artigo apresenta o desenvolvimento da Escala de Generatividade do Casal, versão portuguesa da Couple Generativity Scale de Bertoni, Parise e Iafrate (2012), bem como dados relativos à sua precisão e validade. Este questionário pretende avaliar a capacidade de o casal ultrapassar as fronteiras conjugais e cuidar também dos seus laços familiares e sociais. Com uma amostra de 406 adultos de ambos os sexos que se encontravam numa relação conjugal, foram identificadas qualidades psicométricas que encorajam a utilização da versão portuguesa. Os dados mostram que a estrutura unifatorial original se adequa à presente amostra, que a escala revela elevados níveis de precisão por consistência interna e que se evidenciam relações significativas da generatividade com outras variáveis importantes da conjugalidade, como ter filhos, a conceção da relação e a satisfação conjugal.
\end{abstract}

Palavras-chave: generatividade, casal, satisfação conjugal

\begin{abstract}
The importance of couple relationship in adult life justifies the study of factors that may contribute to its quality. This article presents the development of the Couple Generativity Scale, the Portuguese version of the Couple Generativity Scale by Bertoni, Parise and Iafrate (2012)", as well as data on its accuracy and validity. This questionnaire aims to assess the couple's ability to cross marital boundaries together and to care for their family and social ties. With a sample of 406 adults of both sexes in a marital relationship, good psychometric qualities were found which encourage its use in the Portuguese population. The data show that the original unifactorial structure fits the present sample, that the scale reveals high levels of precision for internal consistency and that significant relationships of generativity with other important variables of conjugality, such as having children, the conception of the relationship and conjugal satisfaction, are evident.
\end{abstract}

Keywords: generativity, couple, marital satisfaction

\footnotetext{
${ }^{1}$ Doutoramento em Psicologia. Professora Auxiliar. Faculdade de Ciências Humanas da Universidade Católica Portuguesa. CRCW Catolica Research Centre for Psychological, Family and Social Wellbeing. Campus Palma de Cima, Palma de Cima, 1649-023 Lisboa, Portugal. Tel.: +351217214 018 Ext.4115. E-mail: susana.costaramalho@ucp.pt

${ }^{2}$ Doutoramento em Psicologia. Professora Associada. Universidade de Lisboa, Faculdade de Psicologia | CICPSI - Centro de Investigação em Ciência Psicológica. Alameda da Universidade, 1649-013 Lisboa, Portugal. Tel.: +351 217943600 . E-mail: ampinto@psicologia.ulisboa.pt

${ }^{3}$ Doutoramento em Psicologia. Professora Associada. Universidade de Lisboa, Faculdade de Psicologia | CICPSI - Centro de Investigação em Ciência Psicológica. Alameda da Universidade, 1649-013 Lisboa, Portugal. Tel.: +351 217943600 . E-mail: mteresaribeiro@psicologia.ulisboa.pt

${ }^{4}$ Doutoramento em Psicologia. Professora Auxiliar. Faculdade de Ciências Humanas da Universidade Católica Portuguesa. CRCW Catolica Research Centre for Psychological, Family and Social Wellbeing. Campus Palma de Cima, Palma de Cima, 1649-023 Lisboa, Portugal. Tel.: +351 968018904. E-mail: mmpedro@fch.lisboa.ucp.pt
}

Revista Iberoamericana de Diagnóstico y Evaluación - e Avaliação Psicológica. RIDEP · No60 · Vol.3 · 71-80 · 2021 ISSN: 1135-3848 print /2183-6051online 


\section{Introdução}

A generatividade é a capacidade de se afastar de uma consciência exclusivamente autocentrada e procurar o cuidar de outros (Erikson, 1963; McAdams, de St Aubin, \& Logan, 1993). Erikson (1963) define-a como a vertente positiva do sétimo estádio do desenvolvimento psicossocial individual afirmando que, para o indivíduo adulto, o resultado mais importante do seu processo de desenvolvimento não se limita à obtenção de bemestar, mas sim à sua capacidade de ser "generativo", criativo e produtivo na relação com os outros e com o mundo. A generatividade inclui, mas não se limita à dimensão biológica procriativa, podendo expressar-se ao nível social através do cuidado às gerações futuras, seja por meio do ensino, apoio ao seu desenvolvimento, envolvimento político ou quaisquer atividades com o propósito de proteção, de promoção da saúde ou do bem-estar do outro. Erikson (1975) aborda o conceito de generatividade como uma orientação ética para o cuidado que marca uma diferença entre a idade adulta e a adolescência. No seu seminal livro Dimensions of a New Identity (1974, p. 124), escreve que depois de os indivíduos aprenderem "o que querem fazer $\mathrm{e}$ quem querem ser" (identidade), e "com quem se importam" (intimidade), estão prontos para aprender "do quê e de quem importa cuidar" (generatividade).

Inspirados nesta teoria, autores contemporâneos propõem que - para lá do desenvolvimento individual - também a relação conjugal integra a dimensão da generatividade, considerando pertinente que se reconheça e estude a capacidade de o casal ultrapassar as fronteiras conjugais e cuidar também dos laços sociais e comunitários. Esta ligação do funcionamento do casal com o conceito de generatividade foi sugerida pelo Modelo Simbólico Relacional (Cigoli \& Scabini, 2006; Scabini \& Cigoli, 2000; Scabini \& Iafrate, 2003), teoria que consideramos particularmente adequada no entendimento da complexidade do relacionamento conjugal. Embora escassos, os estudos sobre esta dimensão têm evidenciado que a generatividade do casal se relaciona positivamente com a confiança entre os cônjuges, a intimidade, o compromisso e o afeto romântico
(Bertoni, Parise, \& Iafrate, 2012), interfere na qualidade parental (McAdams \& Logan, 2004), reflete-se nas atividades partilhadas em casal com o objetivo de melhorar o contexto social ou comunitário (Parise, Gatti, \& Iafrate, 2017), bem como aumenta a satisfação e a vitalidade nas relações (Zinck \& Neel, 2020). Embora Erikson (1963) tenha enfatizado que a parentalidade não é condição necessária nem suficiente para se alcançar a generatividade, encontram-se dados que suportam a ideia de que a experiência da parentalidade se associa com maiores níveis de generatividade (Bertoni, Parise, \& Iafrate, 2012; McAdams \& de St Aubin, 1992; Snarey, Son, Kuehne, Hauser, \& Valliant, 1987; Vaillant \& Milofsky, 1980). Encontra-se ainda suporte empírico para uma diferença entre mulheres e homens no desenvolvimento da generatividade (eg. McAdams \& de St Aubin, 1992; McKeering \& Pakenham, 2000), com as mulheres a evidenciarem níveis mais elevados, sendo possível que as dinâmicas culturais que enfatizam o papel cuidador feminino expliquem estas diferenças.

A Couple Generativity Scale (CGS; Bertoni, Parise, \& Iafrate, 2012) é uma medida breve constituída por sete itens que avalia a generatividade no contexto da relação conjugal, entendida como a capacidade do casal para cuidar dos seus laços familiares e sociais. Não se conhecem estudos em países de língua portuguesa com este instrumento. Foi desenvolvida em Itália a partir das respostas de 1254 participantes de ambos os sexos (16.2\% homens) que se encontravam num relacionamento conjugal, com $(82.1 \%)$ ou sem filhos, provenientes de meios sociais diferentes e grupos étnicos diversificados. A média de idades foi de 48.1 anos ( $\mathrm{SD}=12.79$; amp. 20-80). O estudo original desta escala evidenciou níveis de consistência interna por alfa de Cronbach superiores a .70. A CGS demonstra ter boa validade de construto ao correlacionarse de forma significativa moderada a forte com a dimensão parental e com conceções menos privadas (mais sociais) da relação conjugal em casais sem filhos, e ao não se correlacionar com medidas que envolvem outros construtos diferentes não sobreponíveis, como sejam a conceção da relação conjugal como um assunto privado. 
Em Portugal, considerando o elevado número de separações e divórcios (58\% em 2018, Pordata), considera-se relevante compreender os fatores que - para lá da satisfação - possam contribuir de forma positiva para a qualidade das relações. A adaptação e validação de instrumentos com qualidades psicométricas ajustadas, aplicáveis neste contexto, revela-se assim útil e necessária. $\mathrm{O}$ objetivo do presente artigo consiste em traduzir, adaptar e estudar as características psicométricas da versão portuguesa da Couple Generativity Scale, que designamos de Escala de Generatividade do Casal, de forma a fundamentar empiricamente a sua utilização a nível prático e teórico na realidade nacional. A par dos cuidados na tradução e adequação cultural do conteúdo dos itens, descrevem-se as qualidades métricas em termos de precisão e validade dos resultados.

\section{Método}

\section{Participantes}

A amostra incluiu 406 participantes $(65.8 \%$ mulheres) entre os 22-77 anos ( $M=41,9$; $D P=9,63)$, essencialmente portugueses (98\%). Em termos de escolaridade, a maioria tinha uma licenciatura (47.8\%) ou grau superior (33.8\%), apenas $0.5 \%$ não completaram o ensino secundário. Trabalhavam maioritariamente por conta de outrem (73.4\%), encontrando-se $6.4 \%$ sem trabalho e $3.4 \%$ reformados. O casamento era a relação conjugal mais comum (73.2\%). A duração das relações de casal variou entre menos de um ano e 48 anos, com uma média de 13.8 anos $(D P=10.24)$. Metade dos participantes (49.8\%) tinham mais do que um filho e $23.6 \%$ não tinha nenhum.

\section{Medidas}

Generatividade. A Escala de Generatividade do Casal (EGC), versão portuguesa da Couple Generativity Scale (Bertoni, Parise, \& Iafrate, 2012; adaptada de Loyola Generativity Scale, McAdams \& Dr St. Aubin, 1992), é uma medida breve com sete itens que avalia a perceção da generatividade do casal em indivíduos numa relação conjugal. São exemplo: "Nós estamos empenhados, como casal, com a nossa comunidade" e "Pensamos que a nossa experiência como casal pode ser útil para outras pessoas". Esta escala pode ser cotada de forma simples, somando os itens tipo Likert de 5 pontos (de 1=discordo completamente a 5=concordo completamente). A pontuação na escala varia entre 7 e 35, sendo que pontuações elevadas na escala indicam elevada perceção de generatividade na relação de casal.

Conceção sobre a relação de casal. A forma como o indivíduo descreve o seu casal, particularmente a sua perspetiva sobre as relações com os contextos intergeracionais e sociais, foi avaliada por meio de quatro itens numa escala de 9 pontos (de 1=discordo completamente a $9=$ concordo completamente), usados pelos mesmos autores (Bertoni, Parise, \& Iafrate, 2012) no estudo anteriormente citado. As pessoas podem conceber a sua relação como um assunto privado que envolve apenas os dois parceiros, ou podem reconhecer que o seu casal se integra numa rede de relações sociais mais amplas. São exemplos de itens: "O meu relacionamento é essencialmente um assunto privado entre mim e o meu parceiro", ou "O meu relacionamento é uma questão social, envolvendo também amigos, conhecidos e colegas".

Satisfação conjugal. Os participantes classificaram numa escala de dez pontos (de $0=n a d a$ a $10=$ completamente) o seu nível de satisfação conjugal global através do item único: "Até que ponto se sente satisfeito com a sua relação de casal?”.

Dados sociodemográficos e relacionais. Foi construído um questionário para descrever as características da amostra e analisar diferenças com base nalgumas dessas variáveis. Este questionário incluiu questões como a idade dos participantes, o sexo, os anos de escolaridade, situação face ao emprego, tipo e duração da relação conjugal, número de filhos.

\section{Procedimentos}

Como princípio do processo de tradução e validação da Escala de Generatividade do Casal, obteve-se junto dos autores autorização para utilizar a escala, que nos foi disponibilizada na língua original italiana, bem como na versão inglesa como referente. Para proceder à tradução do instrumento seguiram-se orientações internacionalmente estabelecidas (Hambleton, 2001; Hambleton, Merenda, \& Spielberger, 2005; 
van de Vijver \& Hambleton, 1996) e contou-se com a colaboração de duas tradutoras independentes, uma delas com formação em Psicologia. Uma fez a tradução do italiano para português, tendo a outra realizado a respetiva retroversão para a língua italiana, que foi então comparada com o instrumento original e com a versão inglesa. Quando o resultado da tradução foi considerado concordante procedeu-se a 20 aplicações experimentais com o objetivo de detetar eventuais dificuldades no entendimento dos itens, tendo estas permitido aperfeiçoar nalguns pontos a linguagem e chegar à adequação da tradução final. Os 20 participantes neste estudo piloto apresentaram, no que concerne as suas características sociodemográficas, uma forte similaridade com a presente amostra: $60 \%$ mulheres $(n=12)$, com uma média de idades 42.1 anos $(D P=9.46)$ maioritariamente licenciados ou com grau superior $(80 \%)$. O casamento era a relação conjugal mais comum $(75 \%)$, tendo as relações uma duração média de 14.15 anos ( $D P=9.13)$. Um quarto dos participantes não tinha filhos. As alterações no enunciado dos itens resultantes das aplicações experimentais foram diminutas e resultaram exclusivamente de sugestões ao nível de palavras/expressões (ex. "estamos empenhados" em vez de "estamos comprometidos" e "somos uma referência" em vez de "somos um ponto de referência").

O procedimento de recolha dos dados teve início após aprovação da Comissão de Ética do Centro de Investigação onde se integra a primeira autora, entidade à data responsável pela avaliação científica e ética dos projetos de investigação, no âmbito de um estudo mais vasto sobre as relações de casal. Decorreu online por via da plataforma Qualtrics Survey Software, através de diversas redes sociais num processo bola-de-neve, atingindo assim uma amostra de conveniência não probabilística e não representativa. Definiu-se como critérios de inclusão a idade e o estado civil, exigindo-se como mínimo os 18 anos e o envolvimento atual numa relação conjugal, casamento ou coabitação. Cada questionário era precedido por um termo de consentimento informado dando conhecimento do carácter voluntário, anónimo e confidencial da participação no estudo, bem como da possibilidade de a qualquer momento, sem penalização, abandonar o preenchimento.

\section{Análise dos dados}

A validade fatorial do modelo na amostra em estudo foi avaliada através de uma Análise Fatorial Confirmatória (AFC), com recurso ao software AMOS SPSS v25. Nos estudos realizados na área científica da Psicologia, a AFC é usualmente utilizada como parte do processo de validação de uma escala ou outro instrumento de medição, sendo o seu principal objetivo servir como instrumento de análise da estrutura interna dos dados, enquanto fonte de validade de construto (Pérez, 2020). Deve considerar-se que qualquer modelo AFC é uma aproximação à realidade, constituindo representações de teorias formais sobre os fenómenos do mundo real, cuja construção é levada a cabo por abordagens hipotético-dedutivas sucessivas a tais fenómenos (Pérez, 2020).

Procedeu-se inicialmente à análise dos itens, avaliando-se a sensibilidade da medida com base nos valores de assimetria e curtose, das correlações inter-item e item-total. Considerou-se que valores de assimetria superiores a três e de curtose superiores a sete representariam desvios significativos à normalidade (Marôco, 2014). A utilização destes valores para avaliar a normalidade das variáveis é uma prática frequentemente utilizada e recomendada (IBM SPSS, 2010). As correlações inter-item foram consideradas adequadas quando entre .15 e .50 (Clark \& Watson, 1995) e as correlações itemtotal quando entre .30 e .70 (Nunnally \& Bernstein, 1994).

O modelo foi ajustado estritamente quando necessário, com base em fundamentação teórica e em pressupostos estatísticos, considerando índices de modificação com valores acima de 4 que sugeriram relações entre erros de medida (Hair, Black, Babin, Anderson, \& Tatham, 2006; Marôco, 2014). O modelo foi considerado ajustado aos dados com valores de CFI, TLI e GFI superiores a 0.90 ; o valor de RMSEA inferior a 0.06 ; os valores de $\chi^{2} / \mathrm{df}$ inferiores a 2 ; e os índices AIC e ECVI foram utilizados para comparar os modelos, sendo o modelo com os valores mais baixos aquele que indica melhor ajustamento 
(Arbuckle, 2008; Browne \& Cudeck, 1992; Hu \& Bentler, 1999; Marôco, 2014).

Todos os restantes dados foram analisados utilizando o software SPSS v25 (IBM SPSS, 2017). A fidedignidade foi avaliada através do valor dos alfas de Cronbach para avaliar a consistência interna do questionário. Considerou-se que valores que indicam boa consistência interna se situam acima de .70 (Nunnally, 1978). A validade discriminante foi avaliada com base nos resultados das correlações de Pearson entre a generatividade, a satisfação conjugal, a conceção sobre $\mathrm{o}$ relacionamento de casal e o número de filhos. Realizaram-se análises descritivas das variáveis em estudo, verificando médias e desvio-padrão. Efetuaram-se testes $\mathrm{T}$ com o objetivo de testar diferenças de médias entre grupos em função das variáveis sociodemográficas e relacionais. Usámos como critério para avaliar a magnitude das diferenças o proposto por Cohen (1988), sendo os seguintes valores de referência para interpretação: $.01=$ pequeno efeito, . .06= efeito moderado, .14= efeito alargado.

\section{Resultados}

O primeiro passo na validação da Escala de Generatividade do Casal foi a análise dos itens de forma a verificar a existência de eventuais desvios grosseiros da normalidade (Quadro 1). O item 6 - "Gostaríamos de transmitir os nossos valores e todas as coisas boas que recebemos aos nossos filhos" - apresenta um valor de curtose superior ao valor de referência de sete.

\section{Validade fatorial}

$\mathrm{Na}$ AFC realizada com o objetivo de testar o modelo de medida unifatorial proposto originalmente pelos autores, os valores dos índices de qualidade do ajustamento revelaram um bom ajustamento do modelo aos dados da presente amostra, após eliminação de 4 outliers e correlação de erros sugerida pelos índices de modificação superiores a $4 \quad(\chi 2 / \mathrm{df}=.858$, CFI $=1.00, \quad$ TLI $=1.004, \quad$ GFI $=.995$, RMSEA=.000). O índice AIC baixou de 127.73 para 46.86 e o ECVI de .315 para .116, confirmando o melhor ajustamento deste modelo.
Todos os itens saturam significativamente no fator, com loadings equilibrados e superiores a .50 (cf. fig. 1). Os índices de modificação evidenciam que a correlação entre os erros dos itens 5 e 7 (e5 e $e 7)$ é a que mais contribui para um melhor ajustamento do modelo (M.I.=35.604), seguida da correlação entre os erros dos itens 4 e 6 (M.I.=32.806), do erro do item 2 com os erros dos itens 5,7 e 4 (respetivamente M.I. $=12.854,7.912$, 6.589) e, por último, do erro do item 1 com o erro do item 3 (M.I.=5.276). Analisámos os itens relativamente às suas especificidades e coerência teórica, observando ainda a potencial similitude semântica de alguns.

As correlações sugeridas entre os erros dos itens 5 e 7, assim como entre os erros dos itens 1 e 3, parecem-nos justificadas pela sua similitude semântica: a referência explícita aos amigos, no primeiro caso, e a disponibilidade para os outros, no segundo caso. Relativamente às correlações entre os restantes erros, consideramos que poderão justificar-se pela especificidade teórica da medida de generatividade. Não obstante a unidimensionalidade pensada pelos autores, o conceito de generatividade afigura-se teoricamente complexo e multifacetado, incluindo aspetos como a orientação para o heterocuidado, a "criação criativa" e a produtividade na promoção do bem-estar dos outros e do mundo. Nesta perspetiva, entendemos conceptualmente possível a relação dos erros de medida.
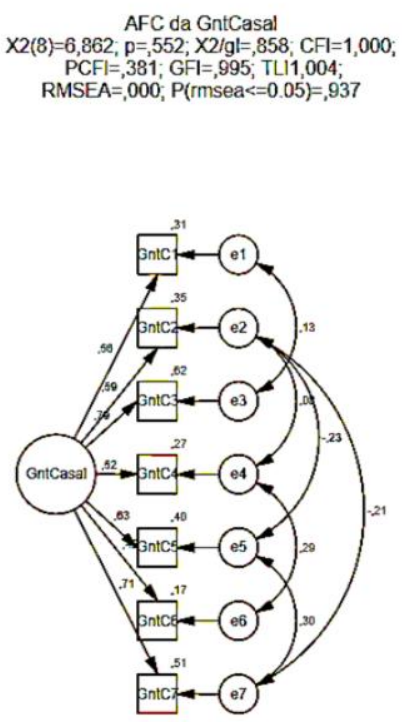

Figura 1. Análise Fatorial Confirmatória da medida de Generatividade do Casal 
Quadro 1. Estatísticas Descritivas dos Itens e do total na Escala de Generatividade do Casal por Amostra Total $(N=406)$

\begin{tabular}{lccccc}
\hline & $\mathrm{M}$ & Mediana & Intervalo & Assimetria & Curtose \\
\hline Item 1 & 3,81 & 4,00 & $1-5$ & -0.59 & 0.26 \\
Item 2 & 4,38 & 5,00 & $2-5$ & -0.94 & -0.04 \\
Item 3 & 4,02 & 4,00 & $1-5$ & -0.80 & 0.08 \\
Item 4 & 4,66 & 5,00 & $2-5$ & -1.90 & 3.36 \\
Item 5 & 3,08 & 3,00 & $1-5$ & -0.24 & -0.39 \\
Item 6 & 4,85 & 5,00 & $1-5$ & -4.70 & 26.78 \\
Item 7 & 3,79 & 4,00 & $1-5$ & -0.53 & 0.09 \\
EGC & 4,08 & 4,14 & $2-5$ & -0.78 & 0.55 \\
\hline
\end{tabular}

Quadro 2. Média, desvio-padrão e resultados das correlações de Pearson entre a medida de Generatividade do Casal e as restantes variáveis em estudo $(N=406)$

\begin{tabular}{llllc}
\hline Variáveis & $M$ & $S D$ & $r$ & $p$ \\
\hline Generatividade do casal & 4.08 & 0.58 & - & - \\
$\mathrm{N}^{\circ}$ de filhos & 1.96 & 0.96 & $.17^{* *}$ & .001 \\
Satisfação conjugal & 8.50 & 1.75 & $.56^{* *}$ & \\
Conceção da relação de casal como um assunto privado & 7.47 & 1.75 & -.06 & .212 \\
Conceção da relação de casal como um assunto de família & 4.36 & 2.68 & $.25^{* *}$ & .001 \\
Conceção da relação de casal como envolvendo amigos e & 4.11 & 2.54 & $.20^{* *}$ & .001 \\
redes informais & 3.51 & 2.51 & $.27^{* *}$ & .001 \\
Conceção da relação como um assunto social & & & &
\end{tabular}

\section{Fidedignidade da medida}

O passo seguinte implicou o cálculo da consistência interna, correlações médias interitens e o leque de correlações item-total corrigidas da Escala de Generatividade do Casal. A avaliação da consistência interna da escala revelou um bom valor do alfa de Cronbach $(\alpha=.80)$. Apesar de o item 6 ter revelado desvios à normalidade, a consistência interna da escala sem este item não apresentava nenhuma melhoria $(\alpha=80)$, pelo que - considerando a sua relevância e o facto de ser este um primeiro estudo com o instrumento em língua portuguesa - optamos por não o retirar. As correlações médias inter-item mantiveram-se dentro dos limites adequados de .15 a .50 (Clark \& Watson, 1995), com exceção para a correlação do item 7 com o item 3 $\left(r=.57^{* *}\right)$ e com o item $5\left(r=.61^{* *}\right)$. O leque de correlações item-total corrigidas apresenta valores adequados entre .40 e .70 (Nunnally \& Bernstein, 1994).

\section{Validade de construto}

A validade de construto da escala em estudo foi avaliada com base nos resultados apresentados no Quadro 2, referentes às correlações entre a medida de Generatividade e as a medidas de conceção do relacionamento de casal, satisfação conjugal e existência de filhos. A Escala de Generatividade do Casal correlacionou-se de forma positiva significativa com o número de filhos, com a satisfação conjugal e com conceções menos privadas (mais sociais) da relação conjugal. Não se observaram correlações significativas com medida que envolve construto diferente não sobreponível, como a conceção da relação conjugal como um assunto privado. Relativamente a esta variável, a perspetiva da relação como um assunto privado entre os dois parceiros $(M=7.47$; $S D=1.75)$ prevalece significativamente sobre as outras visões da relação conjugal.

Por meio da correlação parcial explorámos a relação entre a generatividade e a satisfação conjugal, controlando a variável referente ao número de filhos da relação. Observou-se uma correlação positiva forte (Cohen, 1988), com níveis elevados de generatividade associados a níveis elevados de satisfação conjugal [ $r=.57$, $n=403, p<.001$ ], A verificação da correlação de ordem zero $(r=.56)$ sugere que o controlo da existência e número de filhos teve praticamente efeito nulo sobre a força da relação entre estas duas variáveis.

Explorámos ainda, do mesmo modo, a relação entre a generatividade e a conceção pessoal sobre a relação de casal, controlando o número de filhos da relação. Observam-se correlações positivas fracas (Cohen, 1988), com níveis superiores de generatividade associados a conceções mais sociais da relação conjugal 
$[.21<r<.27, n=403, p<.001]$. A verificação das correlações de ordem zero (Pearson) sugere que o controlo da existência e número de filhos teve praticamente nenhum efeito sobre a força da relação entre as variáveis referidas.

\section{Análises da EGC em função de variáveis sociodemográficas}

Explorámos a existência de diferenças nos resultados da EGC entre mulheres e homens, entre indivíduos casados e coabitantes, assim como entre pessoas com e sem filhos, por meio de testes- $t$ para amostras independentes. Não se observaram diferenças significativas entre mulheres e homens. Evidenciaram-se diferenças significativas entre os indivíduos casados $(M=4.12, \quad S D=.57)$ e coabitantes $\quad[M=3.96$, $S D=.57 ; \mathrm{t}(404)=-2.49, p=.013]$, sendo pequeno o tamanho do efeito (eta squared=.015). Observaram-se diferenças significativas entre os indivíduos com filhos $(M=4.12, S D=.54)$ e sem filhos $[M=3.95, \quad S D=.66 ; \quad \mathrm{t}(141.272)=-2.41$, $p=.017]$. A magnitude da diferença nestas médias foi pequena (eta squared=.014) (Cohen, 1988).

Não se encontra correlação entre o tempo de relação conjugal e os valores da generatividade. $\mathrm{Na}$ mesma linha, não se encontram diferenças nos valores de generatividade quando comparamos pessoas em relação de maior ou menor duração.

\section{Discussão}

O ponto de partida para a presente investigação foi a tradução, adaptação e estudo das propriedades psicométricas da versão portuguesa de uma medida de generatividade nas relações de casal, dimensão que não encontramos em nenhum artigo publicado com amostra portuguesa. Avaliou-se o instrumento Escala de Generatividade do Casal numa amostra de adultos envolvidos numa relação conjugal, designadamente a sua validade fatorial, fidedignidade e validade de construto. $\mathrm{O}$ presente estudo pretendeu ainda avaliar a dimensão medida por este instrumento em função de algumas variáveis sociodemográficas e relacionais.

Os resultados relativos à validade fatorial da escala indicaram que a estrutura unifatorial com sete itens proposta pelos autores (Bertoni, Parise,
$\&$ Iafrate, 2012) obteve um ótimo ajustamento na amostra do presente estudo. Deve ter-se em consideração que, como afirma Pérez (2020), qualquer modelo de análise fatorial confirmatória constitui uma aproximação à realidade, integrando uma representação levada a cabo por abordagens hipotético-dedutivas sobre os fenómenos do mundo real. No estudo com o questionário original, a escala teve boa consistência interna, reportando um coeficiente de alfa de .70 . No presente estudo, o coeficiente alfa de Cronbach foi de .80, informando sobre a excelente fidedignidade da medida na nossa amostra (Nunnally, 1978). O leque de correlações itemtotal corrigidas reforça a fidedignidade dos dados, apresentando valores moderados a elevados entre .40 e .70 (Nunnally \& Bernstein, 1994). As correlações médias inter-item mantiveram-se dentro dos limites adequados de .15 a .50 (Clark \& Watson, 1995), com exceção para a correlação mais elevada do item 7 com o item 3 e com o item 5 (itens mais especificamente centrados no casal enquanto ponto de referência para as pessoas próximas), evidenciado que na generalidade os itens que constituem a EGC cobrem um espectro de questões nem demasiado heterogéneas nem demasiado homogéneas.

A avaliação da EGC quanto à sensibilidade da medida revelou uma adequada distribuição dos resultados, verificando-se que todos os itens exceto um apresentaram valores de simetria e de curtose dentro da distribuição normal. O item 6 ("Gostaríamos de transmitir os nossos valores $e$ todas as coisas boas que recebemos aos nossos filhos.") revelou-se problemático com desvios à normalidade. Optámos, contudo, por não o excluir pelo facto de ser um item bastante relevante na avaliação da generatividade pela sua retirada não aumentar em nada a consistência interna da escala e, ainda, pelo facto de ser este um primeiro estudo com o instrumento em língua portuguesa. Estudos futuros poderão reforçar a avaliação das características psicométricas do instrumento e possibilitar diferentes opções.

Sobre este desvio, sabemos que muito embora um valor discrepante possa resultar dum erro de medida, este pode ser também um resultado genuíno, dando conta de um comportamento extremo da variável (Pino, 2015). Se assim for, este deverá ser estudado e não removido. No 
nosso entender, a formulação do item não é perfeita, nomeadamente a expressão "todas as coisas boas que recebemos”. Já no pré-estudo, em que a formulação inicial era "tudo o que de melhor recebemos", se evidenciaram dúvidas na compreensão do item, assentes sobretudo na compreensão do que se pretende exatamente referir com "estas coisas boas ou melhores de que falamos"? Mais, não será indiferente a dupla entrada do mesmo item, abarcando a transmissão aos filhos quer dos valores quer de outros elementos positivos, estes porventura menos claros. Neste contexto, sugerimos, pois, que em estudos futuros se preste atenção ao comportamento deste item 6 para que se possa aferir sobre a pertinência da sua manutenção na escala.

$\mathrm{Na}$ comparação de grupos, evidenciam-se diferenças significativas na dimensão da generatividade entre indivíduos casados e coabitantes, assim como entre indivíduos com e sem filhos, com os primeiros a pontuar significativamente mais. No que se refere à existência de filhos, muito embora a parentalidade não tenha sido apontada como condição necessária para se alcançar a generatividade (Erikson, 1963), estudos anteriores suportam a ideia de que a experiência da parentalidade se associa com maiores níveis de generatividade (Bertoni, Parise, \& Iafrate, 2012; McAdams and de St Aubin, 1992; Snarey et al., 1987; Vaillant \& Milofsky 1980), o que se verificou também na nossa amostra. Relativamente à diferença entre indivíduos casados e coabitantes, não encontramos dados anteriores que permitam explicar esta diferença. Poderemos colocar a hipótese de que a disposição do casal para ultrapassar as fronteiras conjugais e cuidar de outros laços, sociais e comunitários, se ligue à assunção do contrato social que - por definição - subjaz ao casamento, o que poderá explicar esta diferença. Não encontrámos no presente estudo suporte para a existência de diferenças entre mulheres e homens na dimensão da generatividade (eg. McAdams \& de St Aubin, 1992; McKeering \& Pakenham, 2000).

Relativamente à validade discriminante, a medida de generatividade apresentou correlações positivas, embora fracas (Cohen, 1988), com as conceções mais sociais da relação de casal, não se encontrando qualquer relação da generatividade com conceções mais privadas da relação conjugal, o que vai ao encontro da definição do construto e corrobora o estudo original (Bertoni, Parise, \& Iafrate, 2012). Estes dados reforçam a perspetiva de que olhar a própria relação conjugal como algo que, de alguma forma, envolve outros que estão para além das fronteiras do casal, se associa ao desenvolvimento da generatividade e responsabilidade social (Bertoni, Parise, \& Iafrate, 2012).

Encontrou-se ainda uma correlação muito significativa, ainda que fraca, com o número de filhos, reforçando a procriação como um aspeto importante do conceito de generatividade e informando que a experiência da parentalidade se associa - também na nossa amostra - com níveis superiores de generatividade (Bertoni, Parise, \& Iafrate, 2012; McAdams and de St Aubin, 1992; Snarey et al., 1987). Ter filhos parece assim poder facilitar a adoção de um olhar mais amplo e menos autocentrado, potenciando que os indivíduos se percecionem mais como um recurso para os outros. A elevada correlação da generatividade com a satisfação conjugal informa da ligação entre os dois conceitos e reforça evidências prévias (eg. Zinck \& Neel, 2020) de que a generatividade, a satisfação e a vitalidade nas relações se encontram associadas.

Interessante notar que, relativamente à perspetiva sobre a relação de casal, variável que utilizámos como referente neste estudo, são os casais com filhos que mais consideram a relação de casal como um assunto privado, dados que contrariam estudos anteriores (Bertoni, Parise, \& Iafrate, 2012). Se, como afirmam diversos autores (eg. Canavarro \& Pedrosa, 2005), o nascimento de um filho desencadeia na família movimentos de mudança que passam nomeadamente por uma redefinição de limites perante o exterior, de forma a permitir uma maior abertura da "nova" família às suas famílias de origem e à comunidade, poderemos também hipotetizar que o nascimento dos filhos coloque alguns indivíduos numa perspetiva mais fechada e protetora do núcleo familiar, procurando eventualmente defender-se de avaliações externas que se assumem como comuns - por exemplo, sistemas familiar e escolar em permanente comunicação e recíproca vigilância (eg. Relvas, 1996). Esta hipótese 
parece-nos consistente com a perspetiva de García-Méndez, Rivera-Aragón, Reyes-Lagunes e Díaz-Loving (2006), ao enfatizarem como a família se move permanentemente num continuum que inclui olhares, dinâmicas e padrões de interação que facilitam ou dificultam o movimento e a mudança. Por último, quando comparados com indivíduos coabitantes, os indivíduos casados evidenciam uma conceção mais social da relação, dado que nos parece consistente com a ideia de aqueles que escolhem casar valorizarem, à partida, a dimensão social (contrato) do compromisso conjugal.

O presente estudo revela um conjunto de limitações que importa assinalar. Em primeiro lugar, a amostra em estudo envolveu dois terços de mulheres e três quartos de indivíduos casados. Neste sentido, os resultados que apresentamos devem ser cuidadosamente utilizados, considerando a heterogeneidade em relação a estas variáveis sociodemográficas. Por outro lado, a amostra foi recolhida por conveniência, não sendo como tal necessariamente representativa da população portuguesa. Outra limitação diz respeito ao facto de se estudarem indivíduos e não casais, o que - tratando-se de uma medida que avalia a generatividade do casal - traria decerto dados relevantes. Relativamente aos procedimentos técnicos de validação do instrumento, de futuro será importante complementar o processo através de outros procedimentos, como sejam a validação cruzada com outras amostras e a validade teste-reteste. A validação de um instrumento é efetivamente um processo sempre inacabado (Nunnally \& Bernstein, 1994).

Não obstante as limitações apontadas, foi possível evidenciar as adequadas propriedades psicométricas da versão portuguesa da Escala de Generatividade do Casal, justificando a sua utilização na realidade nacional, seja no âmbito clínico seja na investigação. Paralelamente, os resultados do presente estudo reforçam a ideia de que a generatividade se constitui como uma dimensão importante na relação de casal, associando-se, mas indo para além, de medidas mais comuns como a satisfação conjugal. Como afirma Erikson (1963), faz sentido considerar que, para o indivíduo adulto, o resultado mais importante do seu processo de desenvolvimento não se limita à obtenção de bem-estar ou satisfação, mas alarga-se à sua capacidade de ser "generativo", criativo e produtivo na relação com os outros e com o mundo. Com o presente estudo, reforça-se esta ideia - aqui alargada à dimensão do indivíduo no contexto da sua relação conjugal validando-se a importância de o casal ultrapassar as fronteiras conjugais e cuidar dos laços sociais e comunitários.

\section{Referências}

Arbuckle, J. (2008). Amos 17.0 user's guide. SPSS Inc.

Bertoni, A., Parise, M., \& Iafrate, I. (2012). Beyond satisfaction: Generativity as a new outcome of couple functioning. In P. E. Esposito \& C. I. Lombardi (Eds.), Marriage: Psychological implications, social expectations, and role of sexuality (pp. 115132). New York: Nova Science Publisher.

Browne, M. W., \& Cudeck, R. (1992). Alternative ways of assessing model fit. Sociological methods \& research, 21(2), 230-258.

Canavarro, M. C., \& Pedrosa, A. A. (2005). Transição para a parentalidade: Compreensão segundo diferentes perspetivas teóricas. In I. Leal (Eds.), Psicologia da Gravidez e da Parentalidade (pp. 225-255). Lisboa: Fim de Século.

Cigoli, V., \& Scabini, E. (2006). Family identity: Ties, symbols and transitions. New York: Erlbaum.

Clark, L., \& Watson, D. (1995). Constructing validity: Basic issues in objective scale development. Psychological Assessment, 7(3), 309-319. https://doi.org.10.1037/14805-012

Cohen, J. (1988). Statistical power analysis for the behavioral sciences. Hillsdale, $\mathrm{NJ}$ : Erlbaum.

Erikson, E. H. (1963). Childhood and Society. New York: W.W. Norton \& Company.

Erikson, E. H. (1974). Dimensions of a new identity. New York, NY: Norton.

Erikson, E. H. (1975). Life history and the historical moment. New York, NY: Norton.

García-Méndez, M., \& Rivera-Aragón, S., ReyesLagunes, I., \& Díaz-Loving, R. (2006). 
Construcción de una escala de funcionamiento familiar. Revista Iberoamericana de Diagnóstico y Evaluación - e Avaliação Psicológica, 2(22), 91-110.

Hair, J. F., Black, W. C., Babin, B. J., Anderson, R. E., \& Tatham, R. L. (2006). Multivariate Data Analysis (6 ${ }^{\mathrm{a}}$ ed.). Upper Saddle River, NJ: Pearson Prentice Hall.

Hambleton, R. K. (2001). The next generation of the ITC Test Translation and Adaptation Guidelines. European Journal of Psychological Assessment, 17(3), 164. https://doi.org.10.1027//1015-5759.17.3.164

Hambleton, R.K, Merenda, P., \& Spielberger, C. (2005). Adapting educational and psychological tests for cross-cultural assessment. Mahwah, NJ: Lawrence Erlbaum Associates.

Hu, L. T., \& Bentler, P. M. (1999). Cutoff criteria for fit indexes in covariance structure analysis: Conventional criteria versus new alternatives. Structural Equation Modeling: A Multidisciplinary Journal, 6(1), 1-55.

iBm SPSS (2017). iBm SPSS Statistics Base 19. Chicago, il: SPSS inc

Marôco, J. (2014). Análise estatística com o SPSS Statistics $\left(6^{\text {a }}\right.$ ed. $)$. Pêro Pinheiro: Report Number.

McAdams, D. P., \& de St Aubin, E. (1992). A theory of generativity and its assessment through self-report, behavioral acts and narrative themes in autobiography. Journal of Personality and Social Psychology, 62, 1003$1015 . \quad$ https://doi.org.10.1037/00223514.62.6.1003

McAdams, D. P., de St Aubin, E. D., \& Logan, R. L. (1993). Generativity among young, midlife, and older adults. Psychology and aging, 8(2), 221. https://doi.org/10.1037/0882-7974.8.2.221

McAdams, D. P., \& Logan, R. L. (2004). What is generativity? In E. de St. Aubin, D. P. McAdams, \& T.-C. Kim (Eds.), The generative society: Caring for future generations (pp. 15-31). American Psychological Association. https://doi.org/10.1037/10622-002

McKeering, H., \& Pakenham, K. I. (2000). Gender and generativity issues in parenting: Do fathers benefit more than mothers from involvement in child care activities? Sex Roles, 43, 459-480.

Nunnally, J. C. (1978). Psychometric theory (2a ed.). New York: McGraw-Hill.

Nunnally, J. C., \& Bernstein, I. H. (1994). Psychometric theory (3rd Ed.). New York: McGraw-Hill.

Parise, M., Gatti, F., \& Iafrate, R. (2017). Religiosity, marital quality and couple generativity in Italian couples belonging to a Catholic spiritual association: A qualiquantitative study. Journal of Religion and Health, 56(5), 1856-1869.

https://doi.org/10.1007/s10943-016-0346-0

Pérez, D. O. (2020). Revisión del Concepto de Causalidad en el Marco del Análisis Factorial Confirmatorio. Revista Iberoamericana de Diagnóstico y Evaluación - e Avaliação Psicológica, 1(54), 103-118.

https://doi.org/10.21865/RIDEP54.1.09

Pino, F. A. (2014). A questão da não normalidade: Uma revisão. Revista de Economia Agrícola, 61(2), 17-33.

Relvas, A. P. (1996). O ciclo da família: Perspectiva sistémica. Porto: Edições Afrontamento.

Scabini, E., \& Cigoli, V. (2000). Il famigliare: Legami, simboli e transizioni. Milano: Raffaello Cortina Editore.

Scabini, E., \& Iafrate, R. (2003). Psicologia dei legami familiari. Bologna: Il Mulino.

Snarey, J., Son, L., Kuehne, V. S., Hauser, S., \& Valliant, G. (1987). The role of parenting in men's psychological development. Developmental Psychology, 23, 593-603.

Vaillant, G. E., \& Milofsky, E. (1980). The natural history of male psychological health: IX. Empirical evidence for Erikson's model of the life cycle. American Journal of Psychiatry, 137, 1348-1359.

Van de Vijver, F., \& Hambleton, R. K. (1996). Translating tests. European Psychologist, 1(2), 89-99.

Zinck, K., \& Neel, J. (2020). A Study of Generative Partnership. Adultspan Journal, 19(1), 22-38. https://doi.org/10.1002/adsp.12085 